\title{
Excess placental soluble fms-like \\ tyrosine kinase 1 (sFlt1) may contribute to endothelial dysfunction, hypertension, and proteinuria in preeclampsia
}

\author{
Sharon E. Maynard, ,,2 Jiang-Yong Min, ${ }^{1,2}$ Jaime Merchan,, ${ }^{1,2}$ Kee-Hak Lim,, ${ }^{2,3}$ Jianyi Li, 2,4 \\ Susanta Mondal, ${ }^{1,2}$ Towia A. Libermann, ${ }^{1,2}$ James P. Morgan,, ${ }^{1,2}$ Frank W. Sellke, ${ }^{2,4}$ \\ Isaac E. Stillman, ${ }^{2,5}$ Franklin H. Epstein,, ${ }^{1,2}$ Vikas P. Sukhatme, ${ }^{1,2}$ \\ and S. Ananth Karumanchi ${ }^{1,2}$
}

${ }^{1}$ Department of Medicine, Beth Israel Deaconess Medical Center, Boston, Massachusetts, USA
${ }^{2}$ Harvard Medical School, Boston, Massachusetts, USA
${ }^{3}$ Department of Obstetrics and Gynecology,
${ }^{4}$ Department of Surgery, and
${ }^{5}$ Department of Pathology, Beth Israel Deaconess Medical Center, Boston, Massachusetts, USA

Preeclampsia, a syndrome affecting $5 \%$ of pregnancies, causes substantial maternal and fetal morbidity and mortality. The pathophysiology of preeclampsia remains largely unknown. It has been hypothesized that placental ischemia is an early event, leading to placental production of a soluble factor or factors that cause maternal endothelial dysfunction, resulting in the clinical findings of hypertension, proteinuria, and edema. Here, we confirm that placental soluble fms-like tyrosine kinase 1 (sFlt1), an antagonist of VEGF and placental growth factor (PlGF), is upregulated in preeclampsia, leading to increased systemic levels of sFlt 1 that fall after delivery. We demonstrate that increased circulating sFlt1 in patients with preeclampsia is associated with decreased circulating levels of free VEGF and PIGF, resulting in endothelial dysfunction in vitro that can be rescued by exogenous VEGF and PlGF. Additionally, VEGF and PIGF cause microvascular relaxation of rat renal arterioles in vitro that is blocked by sFlt1. Finally, administration of sFlt1 to pregnant rats induces hypertension, proteinuria, and glomerular endotheliosis, the classic lesion of preeclampsia. These observations suggest that excess circulating sFlt1 contributes to the pathogenesis of preeclampsia.

J. Clin. Invest. 111:649-658 (2003). doi:10.1172/JCI200317189.

\section{Introduction}

The circulating factor secreted by the placenta and the cause of the widespread endothelial dysfunction in preeclampsia has not yet been identified. Several candidates have been suggested, including homocysteine, TNF- $\alpha$, soluble Fas ligand, anti-phospholipid antibodies, and oxidized lipid products, but none of these have been confirmed unequivocally in subsequent work (1, 2). Recently, neurokinin $B$ was reported to be elevated in preeclampsia. Neurokinin B led to transient hypertension when administered intravenously to rats (3);

Received for publication October 18, 2002, and accepted in revised form December 10, 2002.

Address correspondence to: S. Ananth Karumanchi, Beth Israel Deaconess Medical Center, Renal Division, Dana 517, 330 Brookline Avenue, Boston, Massachusetts 02215, USA. Phone: (617) 667-1018; Fax: (617) 667-7843; E-mail: sananth@caregroup.harvard.edu.

Conflict of interest: Vikas P Sukhatme is a consultant and equity holder in Ilex Oncology.

Nonstandard abbreviations used: soluble fms-like tyrosine kinase 1 (sFlt1); placental growth factor (PlGF); human umbilical vein endothelial cells (HUVEC); periodic acid Schiff (PAS); hemolysis, elevated liver function tests, and low platelets (HELLP). however, endothelial dysfunction and proteinuria were not reported. In an attempt to identify novel secreted factors playing a pathologic role in preeclampsia, we performed gene expression profiling of placental tissue from women with and without preeclampsia using Affymetrix U95A microarray chips and found soluble fms-like tyrosine kinase 1 (sFlt1) mRNA (GenBank accession number U01134) to be upregulated in preeclamptic placentas (data not shown). sFlt1, a splice variant of the VEGF receptor Flt1 lacking the transmembrane and cytoplasmic domains, acts as a potent VEGF and PlGF antagonist $(4,5)$. It is produced by a number of tissues, including the placenta $(6,7)$, but its physiologic role is unclear. Recently, both placental sFlt1 expression (8) and sFlt1 levels in the amniotic fluid (9) have been noted to be elevated in preeclampsia; however, systemic levels of sFlt 1 in preeclampsia have not yet been reported.

There is circumstantial evidence that antagonism of VEGF may have a role in hypertension and proteinuria. VEGF is a well-known promoter of angiogenesis; it also induces nitric oxide and vasodilatory prostacyclins in endothelial cells, suggesting a role in decreasing vascular tone and blood pressure $(10,11)$. VEGF has been 
implicated in glomerular healing, and anti-VEGF compounds have been found to increase apoptosis, impair glomerular capillary repair, and increase proteinuria in a rat model of mesangioproliferative nephritis (12). Furthermore, exogenous VEGF was found to accelerate renal recovery in rat models of glomerulonephritis and experimental thrombotic microangiopathy $(13,14)$. More recently, exogenous VEGF was shown to ameliorate post-cyclosporine-mediated hypertension, endothelial dysfunction, and nephropathy (15). Finally, in recent antiangiogenic clinical trials, VEGF signaling inhibitors have resulted in hypertension and proteinuria (16). Collectively, these data suggest that VEGF is important not only in blood pressure regulation but also in maintaining the integrity of the glomerular filtration barrier. We therefore hypothesized that excess circulating sFlt 1 secreted by the placenta in preeclampsia leads to endothelial dysfunction, hypertension, and proteinuria by antagonizing circulating VEGF and PlGF. Here, we demonstrate that excess sFlt1 in patients with preeclampsia causes endothelial dysfunction and produces a syndrome of nephrotic-range proteinuria, hypertension, and glomerular endotheliosis when administered exogenously to animals. Although the primary trigger for abnormal placental development and excess sFlt 1 production in preeclampsia remains speculative, our work suggests that excess sFlt 1 alone may be sufficient to produce generalized endothelial dysfunction and some of the clinical phenotype noted in preeclampsia.

\section{Methods}

Reagents. Human VEGF, rat VEGF, human PlGF, mouse PlGF, recombinant human sFlt1-Fc, and mouse sFlt1Fc were purchased from R\&D Systems (Minneapolis, Minnesota, USA). Human VEGF, human PlGF, human sFlt1, mouse sFlt1, and mouse sFlk1 ELISA kits were also purchased from R\&D.

Patients. Preeclampsia was defined by (1) systolic blood pressure of more than $140 \mathrm{mmHg}$ and diastolic blood pressure of more than $90 \mathrm{mmHg}$ after 20 weeks' gestation in a previously normotensive patient (2), newonset proteinuria (>300 $\mathrm{mg}$ of protein in a 24 -hour urine collection or a random urine protein/creatinine ratio of >0.3), and (3) resolution of hypertension and proteinuria by 12 weeks postpartum. Patients with baseline hypertension, proteinuria, or renal disease were excluded. For the purposes of this study, patients were divided into groups with mild and severe preeclampsia on the basis of the recently published ACOG criteria (17). Healthy, normotensive pregnant women (the "normal" group) were included as controls; six patients with preterm deliveries (the "preterm" group) for other reasons were included as additional controls. Placental samples were obtained immediately after delivery. Four random samples were taken from each placenta, and RNA isolation was performed using Qiagen RNAeasy Maxi Kit (Qiagen, Valencia, California, USA). Serum was collected from pregnant patients at the time of delivery $(t=0)$ and 48 hours after delivery $(t=48)$ after obtaining informed consent. These experiments were approved by the institutional review board at the Beth Israel Deaconess Medical Center.

Northern blots, ELISA, and Western blots. Northern blot experiments were done as previously described (18). The Flt1 probe used for Northern blots was a 500-bp fragment in the coding region from pUC 118 human Flt1 cDNA (a gift from D Mukhopadhyay) and GAPDH cDNA was used as a normalization control. ELISA for human sFlt1, human VEGF, and human PlGF was performed according to the manufacturer's instructions (R\&D Systems). Briefly, the various samples for ELISA measurement were diluted in $0.1 \%$ BSA/Tris-buffered saline and were incubated in a 96-well plate precoated with a capture antibody directed against VEGF, PlGF, or sFlt 1 for 2 hours. The wells were then washed three times in 0.05\%Tween 20/PBS and incubated with a secondary antibody against VEGF, PlGF, or sFlt1 conjugated to horseradish peroxidase for an additional 2 hours. The plates were then washed again three times, substrate solution containing $\mathrm{H}_{2} \mathrm{O}_{2}$ and tetramethylbenzidine was added, and optical density was determined at $450 \mathrm{~nm}$. All assays were done in duplicate, and the protein levels were calculated using a standard curve derived from known concentrations of the respective recombinant proteins. Western blots for PlGF expression in rat blood specimens were performed using a goat polyclonal antibody directed against mouse PlGF-2 (R\&D Systems) using previously described methodology (18). Western blots and ELISA were used for verifying the expression of adenoviral-infected transgenes in the rat plasma as described elsewhere (19).

Endothelial tube assay. Growth factor-reduced Matrigel $(7 \mathrm{mg} / \mathrm{ml}$; Collaborative Biomedical Products, Bedford, Massachusetts, USA) was placed in the wells (100 $\mu \mathrm{l}$ per well) of a prechilled 48 -well cell-culture plate and incubated at $37^{\circ} \mathrm{C}$ for 30 minutes to allow polymerization. Human umbilical vein endothelial cells (HUVEC) (30,000 cells in $300 \mu \mathrm{l}$ of endothelial basal medium with no serum; Clonetics, Walkersville, Maryland, USA) were treated with $5 \%$ patient serum, plated onto the Matrigel-coated wells, and incubated at $37^{\circ} \mathrm{C}$ for 12-16 hours. Tube formation was then assessed through an inverted phase-contrast microscope at $\times 4$ (Nikon Corporation, Tokyo, Japan), and tube length was quantitatively analyzed using the Simple PCI imaging analysis software (Compix Inc. Imaging Systems, Township Pennsylvania, USA).

Renal microvascular reactivity experiments. Microvascular reactivity experiments were done as described previously (20) using rat renal microvessels (internal diameter, $70-170 \mu \mathrm{m})$. In all experimental groups, the relaxation responses of kidney microvessels were examined after precontraction of the microvessels with U46619 (thromboxane agonist) to $40-60 \%$ of their baseline diameter at a distending pressure of 40 $\mathrm{mmHg}$. Once the steady-state tone was reached, the 
responses to various reagents such as VEGF, PlGF, and sFlt1 were examined in a standardized order. All drugs were applied extraluminally.

Animal model. Both pregnant and nonpregnant Sprague-Dawley rats were injected with $1 \times 10^{9} \mathrm{PFU}$ of adenoviruses (Ad Fc, Ad sFlt1, or Ad sFlk1-Fc) by injection into the tail vein. These adenoviruses have been described elsewhere (19) and were generated at the Harvard Vector Core Laboratory. For the low-dose sFlt1 experiment, $1 \times 10^{8} \mathrm{PFU}$ of adenovirus expressing sFlt 1 was used. Pregnant rats were injected on day 8 or 9 of pregnancy (early second trimester), and blood pressure was measured on day 16 or 17 of pregnancy (early third trimester). In nonpregnant animals, blood pressures were measured on day 8 after injection of the adenoviruses. Blood pressures were measured in the rats after anesthesia with sodium pentobarbital $(60 \mathrm{mg} / \mathrm{kg}$ intraperitoneally). The carotid artery was isolated and cannulated with a 3-Fr high-fidelity microtip catheter connected to a pressure transducer (Millar Instruments, Houston, Texas, USA). Blood pressure was recorded and averaged over a 10 -minute period. Blood, tissue, and urine samples were then obtained before euthanasia. We measured plasma levels on the day of blood pressure measurement (day 8 after injection of the adenoviruses), recognizing that 7-10 days after adenoviral injection corresponds to the peak level of expression of these proteins. Circulating sFlt1 and sFlk1 levels were confirmed initially by Western blotting (19) and then quantified using commercially available murine ELISA kits (R\&D Systems). Urinary albumin was measured by standard dipstick and quantified by competitive enzyme-linked immunoassay as has been described elsewhere (21). Urinary creatinine was measured by a picric acid colorimetric procedure kit (Sigma-Aldrich, St. Louis, Missouri, USA).

Statistical comparisons. Results are presented as means \pm SEM, and comparisons between multiple groups were made using ANOVA. Significant differences are reported when $P<0.05$.

Histology and electron microscopy. Harvested kidneys from the rats were placed in Bouin's solution, paraffin embedded, sectioned, and stained with $\mathrm{H} \& \mathrm{E}$, periodic acid Schiff (PAS), or Masson trichrome stain. For electron microscopy, renal tissue was fixed in glutaraldehyde and embedded in araldite-epon mixture; $1-\mu \mathrm{m}$ sections were cut, stained with methylene blue, and assessed before ultrastructural study. Immunofluorescence for fibrin deposits within the glomeruli was done using polyclonal anti-fibrin antibody (ICN Biochemicals, Aurora, Ohio, USA).

\section{Results}

Elevated placental sFlt1 production in preeclampsia. We first confirmed increased placental sFlt 1 mRNA by Northern analysis and found that both sFlt1 and Flt1 messages were upregulated in preeclamptic placentas (Figure 1a). We then measured maternal total sFlt1 serum levels by ELISA in 32 pregnant women with and with- out preeclampsia (Figure 1b). The average serum level of sFlt 1 was almost five times higher in patients with severe preeclampsia than in normotensive pregnant women. To exclude the possibility that this effect was due to the earlier gestational age of patients with preeclampsia, we also measured sFlt1 levels in gestational age-matched normotensive women delivering prematurely for other reasons and found no significant difference between this group and normotensive women with term pregnancies (Figure 1b).

Decreased free VEGF and free PlGF in patients with preeclampsia. sFlt 1 is known to antagonize the proangiogenic molecules VEGF and PIGF by binding to them and preventing their interaction with their cell-surface receptors, Flt1 and KDR. We hypothesized that in preeclampsia, excess sFlt1 causes widespread endothelial dysfunction by interfering with the normal physiologic effects of VEGF and/or PIGF. If so, decreased levels of "free" or unbound VEGF and PIGF should correlate with clinical disease. We measured free VEGF and PlGF serum levels using commercially available ELISA kits that have previously been shown to measure
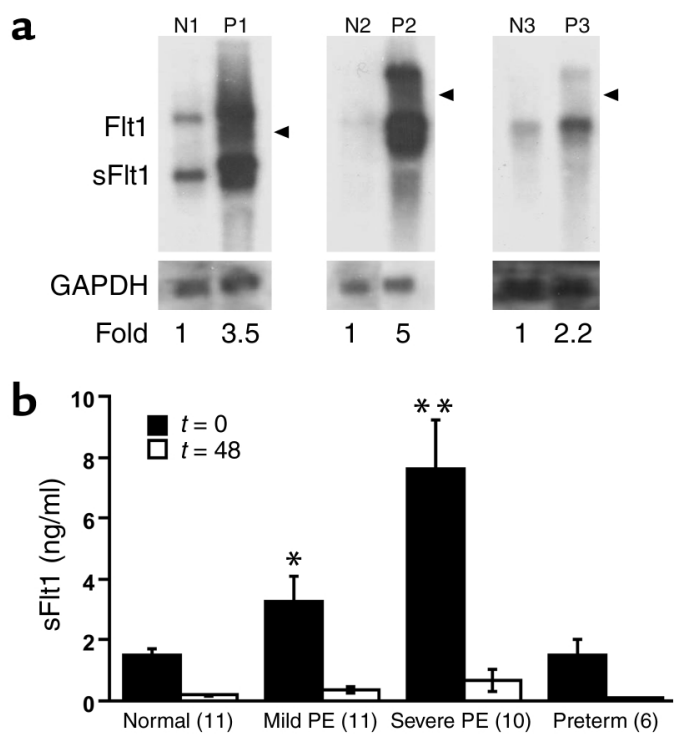

\section{Figure 1}

mRNA and protein expression of sFlt1 in preeclampsia. (a) mRNA expression of placental $s$ Flt 1 from three patients with preeclampsia ( $11, \mathrm{P} 2$, and $\mathrm{P} 3)$ and three normotensive term pregnancies (N1, N2, and N3) were determined by Northern blot analysis. The higher band ( $7.5 \mathrm{~kb})$ is the full-length Flt1 mRNA, and the lower, more abundant band $(3.4 \mathrm{~kb})$ is the alternatively spliced sFlt1 mRNA. GAPDH is included as a loading control, and the location of $28 \mathrm{~S}$ is indicated by an arrowhead. Patients P1 and P2 had severe preeclampsia, whereas patient $\mathrm{P} 3$ had mild preeclampsia. (b) ELISA was performed for sFlt1 on serum from patients with mild preeclampsia (PE), severe preeclampsia and from normotensive pregnant women at term (normal) as described in Table 1. Patients with preterm deliveries were included as additional controls to rule out changes due to gestational age. The numbers of patients tested are shown in parentheses on the $x$-axis. Serum samples were collected before delivery $(t=0)$ and 48 hours after delivery $(t=48)$. ${ }^{*} P<0.05$ and ${ }^{*} P<0.01$ as compared with normotensive controls. 
Table 1

Clinical characteristics of the study patients

\begin{tabular}{|c|c|c|c|c|}
\hline & Normal $(n=11)$ & Mild preeclampsia $(n=11)$ & Severe preeclampsia $(n=10)$ & Preterm $(n=6)$ \\
\hline Maternal age (yrs) & $34.5 \pm 0.9$ & $32.4 \pm 1.6$ & $30.2 \pm 1.3$ & $33.3 \pm 1.5$ \\
\hline Gestational age (wks) & $38.8 \pm 0.2$ & $34.0 \pm 1.0$ & $31.2 \pm 0.9$ & $29.9 \pm 1.7$ \\
\hline Primiparous (\%) & $17 \%$ & $73 \%$ & $70 \%$ & $80 \%$ \\
\hline Systolic blood pressure $(\mathrm{mmHg})$ & $<140$ & $151 \pm 4.3$ & $170 \pm 5.6$ & $<140$ \\
\hline Diastolic blood pressure $(\mathrm{mmHg})$ & $<90$ & $102 \pm 2.6$ & $103 \pm 3.8$ & $<90$ \\
\hline Proteinuria (g protein/g creatinine) & $<0.3$ & $1.1 \pm 0.2$ & $7.0 \pm 1.8$ & $<0.3$ \\
\hline Uric acid $(\mathrm{mg} / \mathrm{dl})$ & NA & $6.4 \pm 0.3$ & $7.0 \pm 0.2$ & NA \\
\hline Hematocrit (\%) & $35.4 \pm 0.6$ & $34.6 \pm 0.9$ & $34.9 \pm 1.5$ & $34.4 \pm 1.4$ \\
\hline Platelet count & $215 \pm 18$ & $214 \pm 34$ & $204 \pm 27$ & $220 \pm 16$ \\
\hline Creatinine $(\mathrm{mg} / \mathrm{dl})$ & $0.6 \pm 0.04$ & $0.6 \pm 0.03$ & $0.5 \pm 0.03$ & 0.5 \\
\hline
\end{tabular}

Values shown are means \pm SEM. Of the six patients in the preterm group, four had preterm labor, one had intrauterine growth retardation, and one had placenta previa. NA, not available.

free VEGF and free $\operatorname{PlGF}(22,23)$. We first confirmed that these ELISA kits measured only unbound VEGF or PlGF by performing a standard curve for VEGF and PlGF proteins in the presence of exogenous recombinant sFlt1. Figure $2(a$ and $b$ ) shows that VEGF and PIGF levels were significantly decreased in the presence of recombinant sFlt 1 . Note that interference of sFlt1 with VEGF measurement was greater than that with PlGF measurement, because Flt 1 binds to VEGF with a higher affinity (VEGF, $K_{d}=10-20 \mathrm{pM}$; PlGF, $\mathrm{K}_{\mathrm{d}}=250$ pM) $(24,25)$. Using these ELISA systems, we then measured free VEGF and free PlGF in the serum of preeclamptic patients and in control women and confirmed that both free VEGF and free PlGF were significantly decreased in patients with preeclampsia (Figure 2, $c$ and d). In fact, the decrease in levels of free VEGF and PlGF was proportionate to the rise in serum sFlt1 levels in these patients.

Impaired angiogenesis due to excess sFlt 1 in preeclamptic serum. To address our hypothesis that excess circulating sFlt1 in patients with preeclampsia causes endothelial dysfunction and leads to an antiangio- genic state, we measured endothelial tube formation, an established in vitro model of angiogenesis. The conditions of the tube formation assay were adjusted so that normal HUVEC cells formed tubes only in the presence of exogenous growth factors such as VEGF or PlGF (data not shown). Under these conditions, we found that although serum from normotensive women induced endothelial cells to form regular tube-like structures, serum from those with preeclampsia inhibited tube formation (Figure 3). Notably, by 48 postpartum hours, this antiangiogenic effect had disappeared from the serum, suggesting that the inhibition of tubes noted with the serum from preeclamptic patients was due to a circulating factor released by the placenta. When sFlt 1 was added to normotensive serum at concentrations noted in patients with preeclampsia, tube formation did not occur, mimicking the effects seen with serum from preeclamptic patients. Finally, when exogenous VEGF and PlGF were added to serum from preeclamptic patients, tube formation was restored (Figure 3). These results suggest that the antiangiogenic proper-
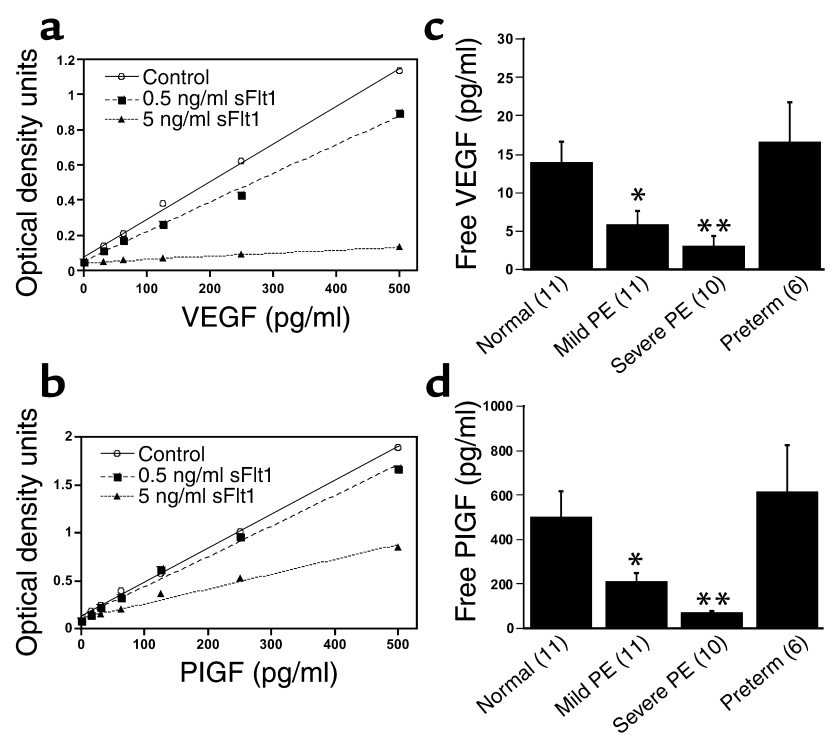

\begin{abstract}
Figure 2
Free VEGF and free PIGF levels are decreased in the serum of patients with preeclampsia. (a) Standard curve for recombinant human VEGF protein was generated in the absence (control) or in the presence of two different doses of recombinant human sFlt1-Fc using the ELISA kit for measurement of human VEGF protein, as described in Methods. (b) Standard curve for recombinant human PIGF protein was generated in the absence (control) or in the presence of two different doses of recombinant human sFlt1-Fc using the ELISA kit for measurement of human PIGF protein, as described in Methods. (c) Free VEGF levels $(\mathrm{pg} / \mathrm{ml})$ at the time of delivery $(t=0)$ were determined by ELISA for the four patient groups described in Figure $1 \mathrm{~b}$ and Table 1. (d) Free PIGF levels $(\mathrm{pg} / \mathrm{ml})$ at the time of delivery were determined by ELISA for the four patient groups described in Figure $1 \mathrm{~b}$ and Table 1. The numbers of patients tested are shown in parentheses on the $x$-axis. ${ }^{*} P<0.05$ and ${ }^{*} P<0.01$ as compared with normotensive controls.
\end{abstract}



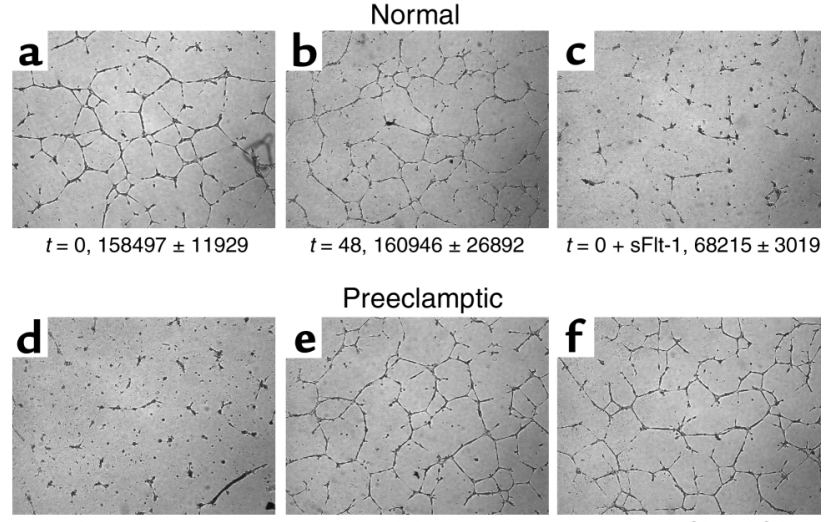

$t=0,87505 \pm 13007$

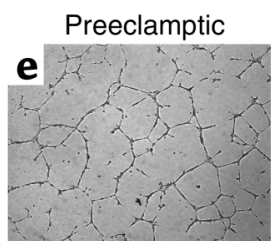

$t=48,237708 \pm 15722$

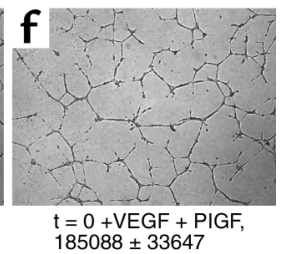

Figure 3

Preeclampsia is an antiangiogenic state due to excess sFlt1. Endothelial tube assay was performed using serum from four normal pregnant controls and four patients with preeclampsia before and after delivery. A representative experiment from one normal control and one patient with preeclampsia is shown. (a) $t=0$ ( $5 \%$ serum from a normal pregnant woman at term). (b) $t=48$ ( $5 \%$ serum from a normal pregnant woman 48 hours after delivery). (c) $t=0$ plus exogenous sFlt1 $(10 \mathrm{ng} / \mathrm{ml})$. (d) $t=0$ (5\% serum from a preeclamptic woman before delivery). (e) $t=48$ ( $5 \%$ serum from a preeclamptic woman 48 hours after delivery). (f) $t=0$ plus exogenous VEGF ( 10 $\mathrm{ng} / \mathrm{ml})$ and PIGF (10 $\mathrm{ng} / \mathrm{ml})$. The tube assay was quantified, and the mean tube length \pm SEM in pixels is given at the bottom of each panel for all the patients analyzed. Recombinant human VEGF, human PIGF, and human sFlt1-Fc were used for the assays.

ties of serum from preeclamptic patients are due to blockade of VEGF and PlGF by endogenous sFlt1. Similar data were also obtained using primary human endothelial cells derived from the uterine microvasculature (data not shown).

Inbibition of VEGF- and PlGF-induced vasodilation by sFlt1. To assess the hemodynamic effects of circulating sFlt1, we performed a series of experiments using an in vitro assay for microvascular reactivity (20). We found that sFlt 1 alone did not cause significant vasoconstriction. However, sFlt 1 blocked the dose-dependent increase in vasodilation induced by VEGF or PIGF (Figure 4a). Data shown in Figure 4b confirmed that sFlt1 significantly inhibited VEGF- and PIGF-induced vasodilation at a level observed in patients with severe preeclampsia. These data suggest that circulating sFlt1 in preeclamptic patients might oppose physiologic vasorelaxation, thus contributing to hypertension.

In vivo effects of sFlt1 on blood pressure and proteinuria. On the basis of these results, we predicted that exogenous sFlt 1 might produce hypertension and proteinuria in an animal model. Adenovirus expressing sFlt 1 has been shown to produce sustained systemic sFlt 1 levels associated with significant antitumor activity (19). This recombinant adenovirus encoding the murine sFlt1 gene product was injected into the tail vein of pregnant rats on day 8 or 9 of pregnancy (normal rat gestation, 21 days). Adenovirus encoding murine $\mathrm{Fc}$ protein in equivalent doses was used as a control to rule out non- specific effects of adenoviruses. Flk1 (VEGF receptor2) has been shown to bind VEGF but not PlGF (24). Hence, adenovirus encoding murine sFlk1-Fc transgene (soluble fusion protein of mouse VEGF receptor Flk1 ectodomain and mouse Fc protein) was chosen as an additional control to determine if the antagonism of VEGF alone would be sufficient to produce a phenotype. We measured intra-arterial blood pressure in the early third trimester (day 16 or 17), corresponding to the onset of hypertension in preeclampsia. These experiments were also completed in nonpregnant female rats to assess whether the effects observed with sFlt1 were dependent on the presence of the placenta.

Blood pressure and albuminuria in the different experimental groups are shown in Table 2 . The mean sFlt1 level in rats injected with adenovirus expressing sFlt1 was $215.5 \pm 81.2 \mathrm{ng} / \mathrm{ml}$, and the mean sFlk1 level was $887.5 \pm 204.8 \mathrm{ng} / \mathrm{ml}$. Pregnant rats treated with sFlt1 had significant hypertension and heavy albuminuria, whereas both Fc and sFlk1-Fc pregnant control rats did not. Nonpregnant rats administered sFlt 1 also developed hypertension and proteinuria. Notably, the sFlk1-Fc-treated nonpregnant rats developed hypertension and proteinuria, whereas the sFlk1-Fc-treated
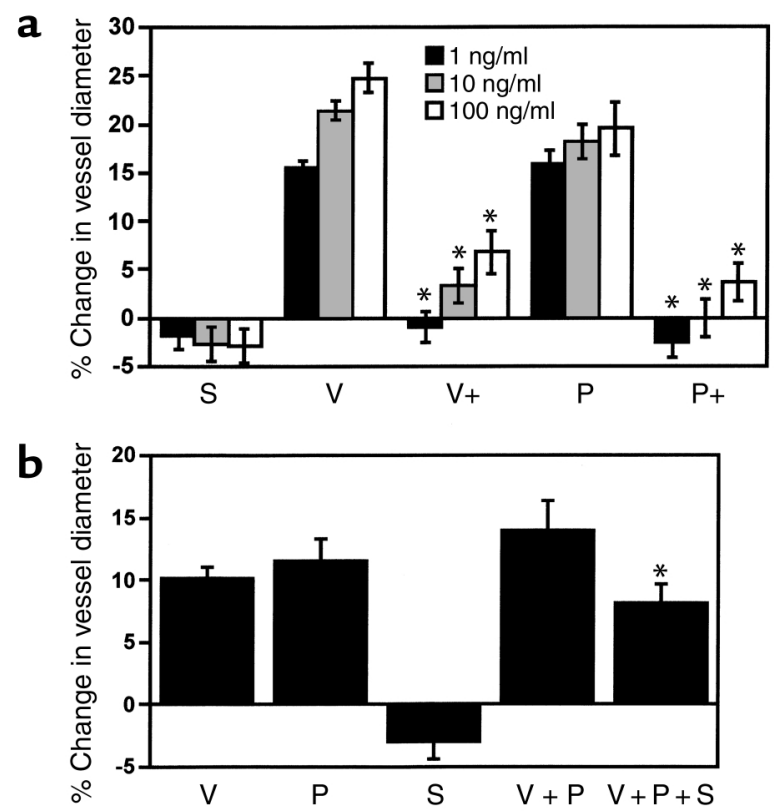

Figure 4

sFlt1 inhibits VEGF- and PIGF-induced vasodilation of renal microvessel. (a) The relaxation responses of rat renal arterioles to sFlt1, VEGF, and PIGF at three different doses were measured. S, sFIt1; V, VEGF; P, PIGF. V+ and P+ represent responses to VEGF and PIGF in the presence of sFlt 1 at $100 \mathrm{ng} / \mathrm{ml}$. A positive change reflects an increase in vessel diameter. (b) Microvascular relaxation responses were measured at physiological doses of VEGF (100 pg/ml), PIGF $(500 \mathrm{pg} / \mathrm{ml}), \mathrm{sFlt} 1(10 \mathrm{ng} / \mathrm{ml}), \operatorname{VEGF}(100 \mathrm{pg} / \mathrm{ml})$ plus PIGF $(500$ $\mathrm{pg} / \mathrm{ml})$, or VEGF $(100 \mathrm{pg} / \mathrm{ml})$ plus PIGF $(500 \mathrm{pg} / \mathrm{ml})$ plus sFlt $1(10$ $\mathrm{ng} / \mathrm{ml})$. Each experiment was repeated in six different rat renal microvessels, and data are reported as means \pm SEM. ${ }^{*} P<0.05$ as compared with VEGF plus PIGF. Reagents used for the assays were recombinant rat VEGF, mouse PIGF, and mouse sFlt1-Fc. 
Table 2

Blood pressure and proteinuria in rats

\begin{tabular}{lccc}
\hline & $n$ & $\begin{array}{c}\text { Mean arterial } \\
\text { pressure }(\mathrm{mmHg})\end{array}$ & $\begin{array}{c}\text { Urine albumin/creatinine } \\
\text { ratio }(\mu \mathrm{gg} / \mathrm{mg})\end{array}$ \\
Fc (pregnant) & 5 & $75 \pm 11$ & $62 \pm 21$ \\
sFlt1 (pregnant) & 4 & $109 \pm 19^{\mathrm{A}}$ & $6923 \pm 658^{\mathrm{B}}$ \\
sFlk1-Fc (pregnant) & 4 & $73 \pm 15$ & $50 \pm 32$ \\
Fc (nonpregnant) & 5 & $89 \pm 6$ & $138 \pm 78$ \\
sFlt1 (nonpregnant) & 6 & $118 \pm 13^{\mathrm{A}}$ & $12947 \pm 2776^{\mathrm{B}}$ \\
sFlk1-Fc (nonpregnant) & 4 & $137 \pm 2^{\mathrm{A}}$ & $2269 \pm 669^{\mathrm{B}}$
\end{tabular}

Pregnant and nonpregnant rats were administered adenovirus expressing Fc (control), sFlt1, or sFlk1-Fc protein. Mean arterial blood pressure (diastolic plus one third of the pulse pressure in $\mathrm{mmHg}$ ) $\pm \mathrm{SEM}$ and mean urine albumin/creatinine ratio (micrograms of albumin per milligram of creatinine) \pm SEM were measured 8 days after adenoviral administration corresponding to the early third trimester in the pregnant rats. ${ }^{A} P<0.05$ and ${ }^{B} P<0.01$ as compared with the control group (Fc). Mean plasma sFlt1 levels were $388 \mathrm{ng} / \mathrm{ml}$ (pregnant) and $101 \mathrm{ng} / \mathrm{ml}$ (nonpregnant) in the sFlt1-treated rats. Mean plasma sFlk1 levels were 775 $\mathrm{ng} / \mathrm{ml}$ (pregnant) and $1000 \mathrm{ng} / \mathrm{ml}$ (nonpregnant) in the sFlk1-Fc-treated rats. up experiments using lower doses of sFlt1. In these rats $(n=5)$, mean serum sFlt1 levels were comparable to those seen in preeclamptic women $(7.3 \pm 3.2 \mathrm{ng} / \mathrm{ml})$. These animals also developed significant hypertension (mean arterial pressure, $119 \pm 5 \mathrm{mmHg}$ ) and albuminuria $(899 \pm 286 \mu \mathrm{g}$ of albumin per milligram of creatinine). Although the degree of albuminuria was much less than that observed in the high-dose experiments (Table 2), it was similar to what was noted in our patients with mild preeclampsia. The pathologic glomerular changes were also suggestive of a less severe phenotype, with endotheliosis and protein resorption droplets occurring in a focal and segmental pattern (Figure 6c). pregnant rats did not. In pregnancy, therefore, the antagonism of VEGF alone appears to be insufficient to produce preeclampsia, possibly owing to high levels of unopposed PlGF secreted by the placenta. In the nonpregnant state, in which PlGF is virtually absent (Figure 5), antagonism of VEGF alone is sufficient to disrupt the balance of pro-and antiangiogenic forces, so as to produce systemic effects.

Renal pathologic changes due to sFlt1. Figure 6a shows the renal lesion that was observed in all sFlt1 treated pregnant rats: glomerular enlargement with occlusion of the capillary loops by swelling and hypertrophy of endocapillary cells (glomerular endotheliosis) (26). No segmental glomerulosclerosis or significant proliferation or vessel wall changes were observed. Electron microscopy of sFlt 1 treated kidneys confirmed the glomerular changes seen on light microscopy (Figure 6b). Extensive capillary occlusion by intraluminal cells with swollen cytoplasm was seen. Podocytes showed protein resorption droplets with only focal foot-process effacement. Immunofluorescence studies of kidneys from sFlt1-treated rats showed focal deposition of fibrin within glomeruli (Figure 6b), changes that have been described as typical of the prepartum stage of human preeclampsia (27). The control Fc-treated rats (Figure 6, a and b) and sFlk1Fc-treated pregnant rats did not show any renal pathology (Figure 6a). This constellation of renal pathologic findings noted in the sFlt1-treated animals is specific and represents the classic findings seen on renal biopsies in human preeclampsia $(26,28$, 29). The sFlt1-treated nonpregnant and sFlk1-Fctreated nonpregnant rats developed renal lesions similar to those in the sFlt1-treated pregnant rats, whereas the Fc-treated nonpregnant rats did not show any renal pathology (data not shown).

Low-dose sFlt1 produces a milder phenotype. Since the sFlt1 levels achieved in our initial experiments were significantly higher than levels observed in humans with preeclampsia (mean of $215.5 \mathrm{ng} / \mathrm{ml}$ in rats vs. $7.6 \mathrm{ng} / \mathrm{ml}$ in humans with severe disease), we conducted follow-

\section{Discussion}

Several conclusions can be drawn from our findings. First, preeclampsia is associated with elevated circulating sFlt 1 protein. It is likely that the excess sFlt 1 production originates in the placenta, since we have shown that placental sFlt1 mRNA is upregulated in preeclampsia and that levels fall within 48 hours after delivery. Second, exogenously administered sFlt 1 is sufficient to produce several of the clinical and pathological findings of preeclampsia in rats, including hypertension and glomerular endotheliosis. Third, the systemic effects of sFlt1 do not require the presence of pregnancy or the placenta, since hypertension and glomerular changes occurred in both nonpregnant and pregnant rats; this suggests a direct effect of sFlt1 on the maternal endothelium. Our work also suggests that sFlt1 acts through its antagonism of both VEGF and PlGF, since the VEGF antagonist sFlk1 did not produce the preeclampsia phenotype in pregnant rats. The observation that nonpregnant rats treated with sFlk1 did develop HTN and proteinuria is consistent with this hypoth-

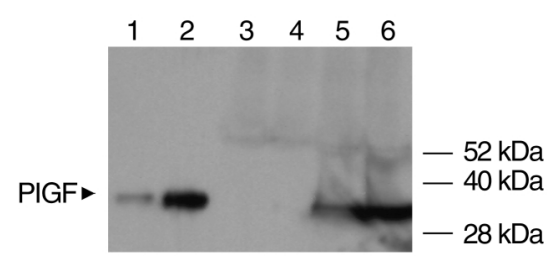

\section{Figure 5}

Western blot analysis for PIGF expression in pregnant rats versus nonpregnant rats. Western blot analysis for PIGF levels in the systemic circulation of rats was performed using blood specimens from two nonpregnant and two pregnant rats (early third trimester) as described in Methods. Lanes 1 and 2 represent $1 \mathrm{ng}$ and $10 \mathrm{ng}$ of recombinant mouse PIGF protein used as a positive control. Twenty microliters of concentrated (10-fold) serum specimens from two nonpregnant rats (lanes 3 and 4) and two pregnant rats in the early third trimester (lanes 5 and 6) were used, and shown is a representative Western blot. The blot shows almost absent PIGF in the nonpregnant rats and expression of PIGF protein in pregnant rats. 
a

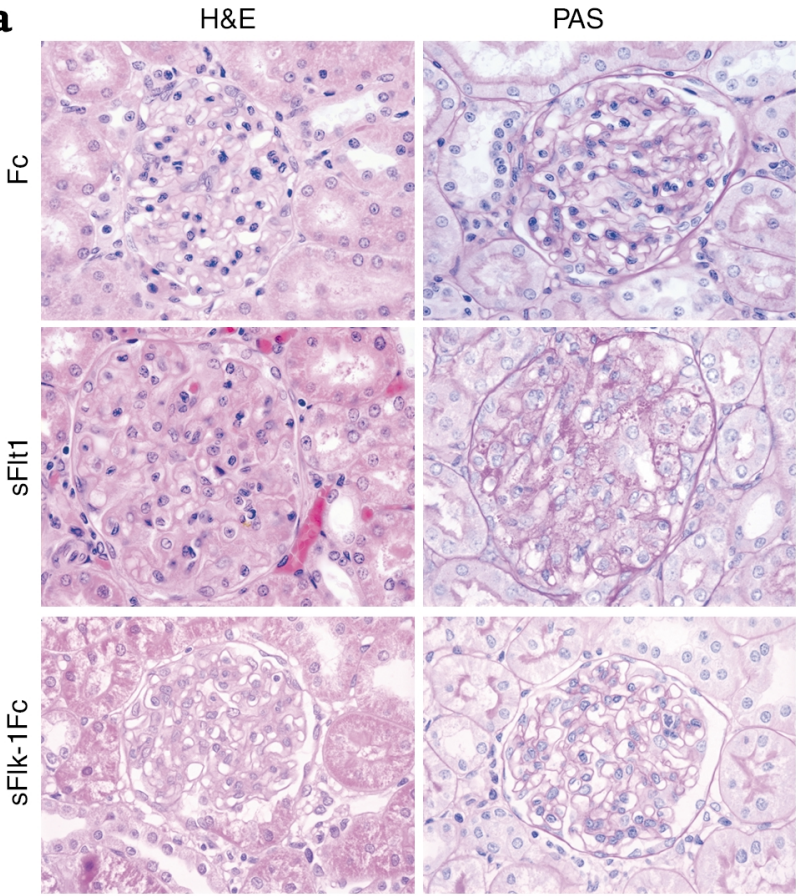

b

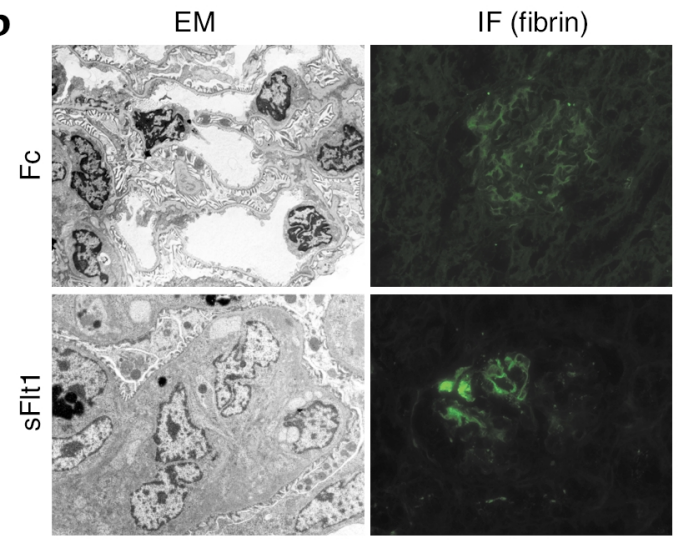

C

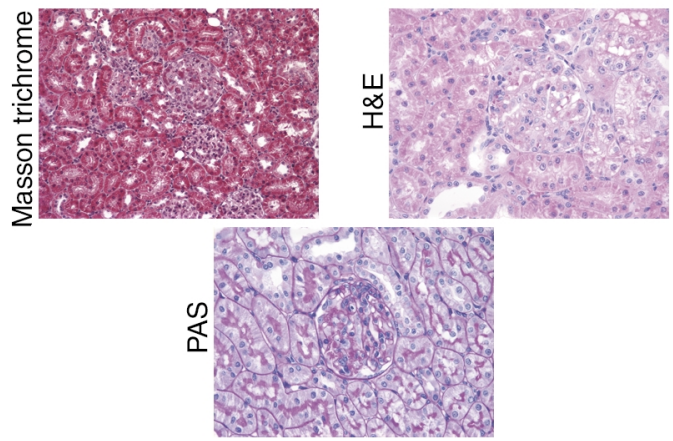

Figure 6

sFlt1 induces glomerular endotheliosis. (a) Histopathological analysis of renal tissue from one representative Fc-treated pregnant rat (upper panel), one sFlt1-treated pregnant rat (middle panel), and one sFlk1-Fc-treated pregnant rat (lower panel) is shown here. H\&E stain shows capillary occlusion in the sFlt1 treated animal with enlarged glomeruli and swollen endothelial cells compared to Fc control animal and sFlk1-Fc control animals. PAS stain of the sFlt1-treated rat demonstrates PAS-negative swollen cytoplasm of endocapillary cells (endotheliosis). Numerous protein resorption droplets are also seen in the PAS section. These pathologic changes are absent in the Fc-treated rat as well as the sFlk1-Fc-treated pregnant rats. All light photomicrographs were taken at $\times 60$ (original magnification). (b) Electron microscopy (EM) and immunofluorescence (IF) for fibrin was performed for the same rats shown in Figure 6a. Electron micrographs of glomeruli from an sFlt1-treated rat (lower panel) confirmed cytoplasmic swelling of the endocapillary cells. There is relative preservation of the podocyte foot processes and the basement membranes. Immunofluorescence for fibrin shows foci of fibrin deposition within the glomeruli of sFlt 1 -treated rats but not Fc-treated rats. The immunofluorescence pictures were taken at $\times 40$ and the electron micrographs were taken at $\times 2400$ (original magnification). All figures are reproduced at identical magnifications. (c) Histopathological analysis of one representative nonpregnant rat treated with low-dose sFlt1 is shown here. Low-power $(\times 30$, original magnification) Masson trichrome staining of renal tissue from the low-dose sFlt1-treated rat shows varying glomerular size representing focal endotheliosis. This degree of variation in glomerular involvement was only noted in the low-dose group. Higher-power H\&E staining and PAS staining showed segmental endotheliosis and protein resorption droplets with preservation of basement membranes.

esis, since circulating PlGF is negligible in this setting. Finally, we have developed a novel experimental model resembling human preeclampsia, suitable for exploring both the pathophysiology of preeclampsia and for testing potential therapeutic compounds.

Emerging data on the role of VEGF in proteinuria lends validity to our findings. In a recent abstract describing new conditional knockout mice, reduction of VEGF production by podocytes alone led to massive proteinuria and glomerular endotheliosis (30). Additionally, VEGF-neutralizing antibodies in clinical cancer trials have resulted in proteinuria (16). These reports support the hypothesis that VEGF deficiency in the glomerulus, as may occur with excess sFlt1 in preeclampsia, produces proteinuria.

The role of VEGF in preeclampsia has received substantial attention. Several authors have reported increased systemic VEGF levels in women with preeclampsia (31-34) while other authors have reported decreased levels (35-37), as we report here (Figure 2c). In reviewing the methodology of these studies carefully, we found that all studies reporting decreased VEGF (35-37) used a commercially available ELISA kit (R\&D Systems), which, in fact, measures free (unbound) VEGF as previously shown by others $(22,23,38)$. All studies reporting increased VEGF in preeclampsia used either radioimmunoassay or a non-R\&D ELISA system, measuring total (bound and unbound) VEGF (31-34). Under many circumstances, these two entities would be interchangeable. However, in pregnancy, circulating sFlt1 is present at very high levels (the mean sFlt 1 level in normal-term pregnancy was $1.5 \pm 0.2 \mathrm{ng} / \mathrm{ml}$ ) (Figure 1b) as compared with the nonpregnant state, in 
which sFlt 1 levels are relatively low (the mean sFlt 1 level in healthy female volunteers was $0.15 \pm 0.04$ $\mathrm{ng} / \mathrm{ml}$ ) (39). Therefore, in normal pregnancy, and especially in preeclampsia where circulating levels of sFlt1 are extremely high, most VEGF is bound to circulating sFlt1 (40). Free VEGF levels, which more accurately reflect effective circulating VEGF, will thus be substantially lower than total VEGF levels. Similarly, the commercial PIGF ELISA kit from R\&D Systems actually measures unbound (or free) PlGF (22), and other groups using this kit have demonstrated low circulating PIGF $(37,41)$, as we have confirmed here (Figure 2d). Seen in this light, the previously confusing and contradictory literature on VEGF supports our hypothesis that preeclampsia is characterized by normal to high total VEGF levels (perhaps induced by placental hypoxia) but low free VEGF and free PIGF levels, owing to a vast excess of sFlt1.

Several aspects of this work suggest that factors in addition to sFlt 1 are likely to be playing a role in the pathogenesis of preeclampsia. Although serum sFlt1 levels were elevated in most patients with preeclampsia, a subset of patients in the mild preeclampsia group had only slightly elevated levels. Thus, sFlt1 may be causative in most but not all cases of preeclampsia in humans. Thrombocytopenia was not noted in our sFlt1-treated animals (data not shown), though it is invariably present in patients with hemolysis, elevated liver function tests, and low platelets (HELLP) syndrome, a variant of preeclampsia. This suggests that additional factors may be involved in HELLP syndrome. Work is in progress to identify such factors, which may be synergistic with sFlt1. It is interesting to note that the pathologic effects of sFlt1 were dose dependent; rats treated with low-dose sFlt1, with plasma levels similar to those seen in preeclamptic women, generally showed milder renal pathology as compared with rats treated with higher doses of sFlt 1 (Figure $6 \mathrm{c}$ ). A possible explanation is that sFlt1 may be one of several factors elaborated by the placenta that influence the severity of preeclampsia. It is also possible that recombinant adenoviral-linked sFlt 1 has a less potent in vivo activity than endogenous sFlt 1 present in human serum or that more prolonged, sustained levels of sFlt 1 are required to produce severe disease.

Our work leaves many unanswered questions and paths for future work. Our data do not distinguish whether sFlt 1 production by the placenta is a primary or secondary event. Hypoxia has been shown to increase sFlt1 production by placental cytotrophoblasts (22). If placental hypoxia is an early event in preeclampsia, sFlt1 release may occur as a secondary phenomenon. It has been proposed that placental angiogenesis is defective in preeclampsia, as evidenced by failure of the cytotrophoblasts to convert from an epithelial to an endothelial phenotype (referred to as pseudovasculogenesis) and invade maternal spiral arteries (42). It seems plausible that angiogenic molecules such as VEGF, PlGF, and sFlt1 may be impor- tant regulators of early placental development and pseudovasculogenesis. In fact, it has recently been shown that exogenous sFlt 1 inhibits placental cytotrophoblast invasion in vitro (8). Thus, excess placental sFlt 1 , in addition to its direct effect on the maternal endothelium in the third trimester, may also play a more primary role in deranged placental development in preeclampsia. In our sFlt1-treated rats, we did not observe the placental pathologic changes typical of preeclampsia, such as placental infarcts and shallow spiral-artery invasion (data not shown). However, this may reflect the fact that sFlt1 protein was administered in the early second trimester, after spiral-artery invasion had already been established. Future studies in which exogenous sFlt 1 is given earlier in pregnancy (i.e., from the first trimester) should clarify this issue, and the role of sFlt 1 in placental cytotrophoblast differentiation and development should continue to be explored.

Little is known about the regulation of transcription and splicing of Flt1/sFlt1. Alternative splicing has been identified as a key regulatory step in $s F l t 1$ production (6). By Northern analysis, however, it appears that both Flt1 and sFlt1 are proportionally increased in preeclamptic placenta (Figure 1a), suggesting that upregulation of sFlt1 is not occurring at the level of alternative splicing but at the level of transcription or mRNA stability. Future work investigating the regulation of sFlt 1 production may clarify these mechanisms.

Our study has other limitations. Although the rapid decline in sFlt 1 levels after delivery and the upregulation of placental sFlt1 mRNA strongly suggest a placental origin of the sFlt1, future studies (for example, measuring sFlt1 levels in the umbilical vein and artery) are needed to show this conclusively. Tissue levels and activity of VEGF/PlGF signaling are not addressed by this study or other current literature. Studies looking at tissue VEGF or PlGF signaling in preeclampsia should clarify the role of sFlt 1 in the pathogenesis even further. In our measurement of blood pressure, we used anesthetized animals; this is known to have blood pressure-lowering effects. Although sFlt1-treated animals had significantly higher mean arterial pressures as compared with controls, it is possible that noninvasive blood pressure monitoring of these animals might produce a even more dramatic change in blood pressure.

Our findings have important implications both for the diagnosis and therapy of preeclampsia and for the use of VEGF/PIGF signaling inhibitors in other diseases, such as cancer. If sFlt 1 overexpression occurs early in pregnancy, it might serve as a diagnostic marker in patients at high risk for the development of preeclampsia. Currently, there is no specific treatment for preeclampsia, and severe cases often require premature delivery of the infant. If excess sFlt 1 plays a causative role in preeclampsia, antagonizing its effects may ameliorate symptoms. For example, exogenous VEGF and/or PlGF therapy might reverse the endothe- 
lial dysfunction noted in these patients, as suggested in our in vitro angiogenesis assays (Figure 3 ). Therapeutic strategies using small-molecule compounds aimed at shifting the angiogenesis balance in favor of proangiogenic molecules might allow delivery to be safely postponed. For example, nicotine has been shown to have proangiogenic properties by inducing endogenous VEGF (43). Moreover, it is well-known that cigarette smoking is associated with a lower incidence of preeclampsia $(44,45)$. Smoking has also been shown to lower sFlt1 levels in humans (46). Thus, short term use of nicotine in cases of severe preeclampsia might be an effective treatment. This work also has implications for anti-VEGF or anti-PlGF compounds currently undergoing clinical trials for the treatment of cancer and other disorders $(19,47)$. The finding of proteinuria and hypertension in nonpregnant rats treated with antagonists to VEGF and PlGF raises concerns over the safety of these agents in humans, and specific monitoring for renal and vascular side effects should be considered. On the other hand, the occurrence of hypertension and/or proteinuria may signal a shift in systemic angiogenic activity and might correlate with response to therapy.

In summary, our findings suggest that excess placental production of sFlt 1 contributes to the hypertension, proteinuria, and glomerular endotheliosis noted in patients with preeclampsia. Antagonizing or overwhelming endogenous sFlt 1 may be a promising therapeutic approach for these patients. A clearer understanding of sFlt 1 gene regulation and splicing and its role in placental and systemic vascular function may lead to better insights into the pathogenesis, treatment, and prevention of preeclampsia.

\section{Acknowledgments}

We thank B. Sachs and the staff in the Department of Obstetrics at the Beth Israel Deaconess Medical Center for help with patient identification and recruitment. We thank C. Bailey for help with microarrays; R. Mulligan for help with adenoviruses; and S. Fisher, S. Lecker, S. Alper, T. Strom, V. Jha, J. Flier, C. Bianchi, M. Kaynar, T. Hampton, Y. Lin, G. Yeo, T. Ghosh, and L. Tsiokas for helpful discussions. This work was funded by a National Institute of Diabetes and Digestive and Kidney Diseases KO8 award and an American Society of Nephrology-Carl W. Gottschalk Research Scholar award to S. A. Karumanchi; by NIH U24DK058739 center grant to T.A. Libermann; by Beth Israel Deaconess Medical Center seed funds to S. A. Karumanchi and V. P. Sukhatme; and by an ASCO Young Investigator award and the Clinical Investigator Training Program of the Beth Israel Deaconess Medical Center-Harvard/Massachusetts Institute of Technology Health Sciences and Technology in collaboration with Pfizer Inc. to J. Merchan.

1. Roberts, J.M. 2000. Preeclampsia: what we know and what we do not know. Semin. Perinatol. 24:24-28.

2. Roberts, J.M., and Cooper, D.W. 2001. Pathogenesis and genetics of pre-eclampsia. Lancet. 357:53-56.
3. Page, N.M., et al. 2000. Excessive placental secretion of neurokinin B during the third trimester causes pre-eclampsia. Nature. 405:797-800. 4. Kendall, R.L., Wang, G., and Thomas, K.A. 1996. Identification of a natural soluble form of the vascular endothelial growth factor receptor, FLT-1, and its heterodimerization with KDR. Biochem. Biophys. Res. Commun. 226:324-328.

5. Shibuya, M. 2001. Structure and function of VEGF/VEGF-receptor system involved in angiogenesis. Cell Struct. Funct. 26:25-35.

6. He, Y., et al. 1999. Alternative splicing of vascular endothelial growth factor (VEGF)-R1 (FLT-1) pre-mRNA is important for the regulation of VEGF activity. Mol. Endocrinol. 13:537-545.

7. Clark, D.E., et al. 1998. A vascular endothelial growth factor antagonist is produced by the human placenta and released into the maternal circulation. Biol. Reprod. 59:1540-1548.

8. Zhou, Y., et al. 2002. Vascular endothelial growth factor ligands and receptors that regulate human cytotrophoblast survival are dysregulated in severe preeclampsia and hemolysis, elevated liver enzymes, and low platelets syndrome. Am. J. Pathol. 160:1405-1423.

9. Vuorela, P., et al. 2000. Amniotic fluid - soluble vascular endothelial growth factor receptor-1 in preeclampsia. Obstet. Gynecol. 95:353-357.

10. Morbidelli, L., et al. 1996. Nitric oxide mediates mitogenic effect of VEGF on coronary venular endothelium. Am. J. Physiol.. 270:H411-H415.

11. He, H., et al. 1999. Vascular endothelial growth factor signals endothelial cell production of nitric oxide and prostacyclin through flk-1/ KDR activation of c-Src. J. Biol. Chem. 274:25130-25135.

12. Ostendorf, T., et al. 1999. VEGF(165) mediates glomerular endothelial repair. J. Clin. Invest. 104:913-923.

13. Masuda, Y., et al. 2001. Vascular endothelial growth factor enhances glomerular capillary repair and accelerates resolution of experimentally induced glomerulonephritis. Am. J. Pathol. 159:599-608.

14. Kim, Y.G., et al. 2000. Vascular endothelial growth factor accelerates renal recovery in experimental thrombotic microangiopathy. Kidney Int. 58:2390-2399.

15. Kang, D.H., et al. 2001. Post-cyclosporine-mediated hypertension and nephropathy: amelioration by vascular endothelial growth factor. Am. J. Physiol. Renal Physiol. 280:F727-F736.

16. Yang, J.C., et al. 2002. A randomized double-blind placebo controlled trial of bevacizumab (anti-VEGF antibody) demonstrating a prolongation in time to progression in patients with metastatic renal cancer: ASCO meeting abstract. Proc. Am. Soc. Clin. Oncol. 21:A15 (Abstr.).

17. American College of Obstetricians and Gynecologists. 2002. ACOG practice bulletin. Diagnosis and management of preeclampsia and eclampsia. Int. J. Gynaecol. Obstet. 77:67-75.

18. Knebelmann, B., Ananth, S., Cohen, H.T., and Sukhatme, V.P. 1998. Transforming growth factor alpha is a target for the von Hippel-Lindau tumor suppressor. Cancer Res. 58:226-231.

19. Kuo, C.J., et al. 2001. Comparative evaluation of the antitumor activity of antiangiogenic proteins delivered by gene transfer. Proc. Natl. Acad. Sci. USA. 98:4605-4610.

20. Sato, K., Li, J., Metais, C., Bianchi, C., and Sellke, F. 2000. Increased pulmonary vascular contraction to serotonin after cardiopulmonary bypass: role of cyclooxygenase. J. Surg. Res. 90:138-143.

21. Magnotti, R.A., Jr., Stephens, G.W., Rogers, R.K., and Pesce, A.J. 1989. Microplate measurement of urinary albumin and creatinine. Clin. Chem. 35:1371-1375.

22. Hornig, C., et al. 2000. Release and complex formation of soluble VEGFR-1 from endothelial cells and biological fluids. Lab. Invest. 80:443-454.

23. Banks, R.E., et al. 1998. Evidence for the existence of a novel pregnancy-associated soluble variant of the vascular endothelial growth factor receptor, Flt-1. Mol. Hum. Reprod. 4:377-386.

24. Park, J.E., Chen, H.H., Winer, J., Houck, K.A., and Ferrara, N. 1994. Placenta growth factor. Potentiation of vascular endothelial growth factor bioactivity, in vitro and in vivo, and high affinity binding to Flt1 but not to Flk-1/KDR. J. Biol. Chem. 269:25646-25654.

25. Ferrara, N., and Davis-Smyth, T. 1997. The biology of vascular endothelial growth factor. Endocr. Rev. 18:4-25.

26. Gaber, L.W., Spargo, B.H., and Lindheimer, M.D. 1994. Renal pathology in pre-eclampsia. Baillieres Clin. Obstet. Gynaecol. 8:443-468.

27. Kincaid-Smith, P. 1991. The renal lesion of preeclampsia revisited. Am. J. Kidney Dis. 17:144-148.

28. Sheehan, H.L. 1980. Renal morphology in preeclampsia. Kidney Int. 18:241-252.

29. Fisher, K.A., Luger, A., Spargo, B.H., and Lindheimer, M.D. 1981. Hypertension in pregnancy: clinical-pathological correlations and remote prognosis. Medicine (Baltimore). 60:267-276.

30. Eremina, V., et al. The role of VEGF-A in glomerular angiogenesis. 2002. J. Am. Soc. Nephrol. 13:100A. (Abstr.)

31. Baker, P.N., Krasnow, J., Roberts, J.M., and Yeo, K.T. 1995. Elevated serum levels of vascular endothelial growth factor in patients with preeclampsia. Obstet. Gynecol. 86:815-821. 
32. Bosio, P.M., et al. 2001. Maternal plasma vascular endothelial growth factor concentrations in normal and hypertensive pregnancies and their relationship to peripheral vascular resistance. Am. J. Obstet. Gynecol. 184:146-152.

33. Hunter, A., et al. 2000. Serum levels of vascular endothelial growth factor in preeclamptic and normotensive pregnancy. Hypertension. 36:965-969

34. Sharkey, A.M., et al. 1996. Maternal plasma levels of vascular endothelial growth factor in normotensive pregnancies and in pregnancies complicated by pre-eclampsia. Eur. J. Clin. Invest. 26:1182-1185.

35. Lyall, F., Greer, I.A., Boswell, F., and Fleming, R. 1997. Suppression of serum vascular endothelial growth factor immunoreactivity in normal pregnancy and in pre-eclampsia. $\mathrm{Br}$. J. Obstet. Gynaecol. 104:223-228.

36. Reuvekamp, A., Velsing-Aarts, F.V., Poulina, I.E., Capello, J.J., and Duits, A.J. 1999. Selective deficit of angiogenic growth factors characterises pregnancies complicated by pre-eclampsia. Br. J. Obstet. Gynaecol. 106:1019-1022.

37. Livingston, J.C., et al. 2000. Reductions of vascular endothelial growth factor and placental growth factor concentrations in severe preeclampsia. Am. J. Obstet. Gynecol. 183:1554-1557.

38. Anthony, F.W., Evans, P.W., Wheeler, T., and Wood, P.J. 1997. Variation in detection of VEGF in maternal serum by immunoassay and the possible influence of binding proteins. Ann. Clin. Biochem. 34:276-280. 39. Barleon, B., et al. 2001. Soluble VEGFR-1 secreted by endothelial cells and monocytes is present in human serum and plasma from healthy donors. Angiogenesis. 4:143-154.

40. Jelkmann, W. 2001. Pitfalls in the measurement of circulating vascular endothelial growth factor. Clin. Chem. 47:617-623.

41. Torry, D.S., Wang, H.S., Wang, T.H., Caudle, M.R., and Torry, R.J. 1998. Preeclampsia is associated with reduced serum levels of placenta growth factor. Am. J. Obstet. Gynecol. 179:1539-1544.

42. Zhou, Y., Damsky, C.H., and Fisher, S.J. 1997. Preeclampsia is associated with failure of human cytotrophoblasts to mimic a vascular adhesion phenotype. One cause of defective endovascular invasion in this syndrome? J. Clin. Invest. 99:2152-2164.

43. Heeschen, C., et al. 2001. Nicotine stimulates angiogenesis and promotes tumor growth and atherosclerosis. Nat. Med. 7:833-839.

44. Newman, M.G., Lindsay, M.K., and Graves, W. 2001. Cigarette smoking and pre-eclampsia: their association and effects on clinical outcomes. J. Matern. Fetal Med. 10:166-170.

45. Zhang, J., Klebanoff, M.A., Levine, R.J., Puri, M., and Moyer, P. 1999. The puzzling association between smoking and hypertension during pregnancy. Am. J. Obstet. Gynecol. 181:1407-1413.

46. Belgore, F.M., Lip, G.Y., and Blann, A.D. 2000. Vascular endothelial growth factor and its receptor, Flt-1, in smokers and non-smokers. $\mathrm{Br}$. J. Biomed. Sci. 57:207-213.

47. Luttun, A., et al. 2002. Revascularization of ischemic tissues by PlGF treatment, and inhibition of tumor angiogenesis, arthritis and atherosclerosis by anti-Flt1. Nat. Med. 8:831-840. 